\title{
A cooperative Homicidal Chauffeur game
}

\author{
Shaunak D. Bopardikar
}

\author{
Francesco Bullo
}

João Hespanha

\begin{abstract}
We address a pursuit-evasion problem involving an unbounded planar environment, a single evader, and multiple pursuers moving along curves of bounded curvature. The problem amounts to a multi-agent version of the classic homicidal chauffeur problem; we focus on parameter ranges in which a single pursuer is not sufficient to capture the evader. We propose a novel cooperative strategy in which the pursuers move in a daisy-chain formation and confine the evader to a bounded region. The proposed policy is inspired by certain hunting and foraging behaviors of various fish species. We characterize the required number of pursuers and the required value of the evader/pursuers speed ratio for which our strategy is guaranteed to lead to confinement.
\end{abstract}

\section{INTRODUCTION}

The homicidal chauffeur game has been studied in great detail. Proposed originally by Isaacs [1], this problem involves a single pursuer who wants to overrun an evader, both moving with fixed speeds. The pursuer has greater speed but has constraints on its turning radius while the evader can make arbitrarily sharp turns. The evader is said to be captured when the distance between the pursuer and evader becomes less than a specified capture radius. We present a multi-agent homicidal chauffeur problem in which a single pursuer is not sufficient to capture the evader. We present a cooperative strategy for multiple pursuers to confine the evader in a bounded region which the evader cannot leave without being captured.

\section{A. Related Work}

The classical homicidal chauffeur problem was proposed and solved by Isaacs [1]- [2]. This solution gives a condition on the game parameters, i.e., the speed ratio of the players, the capture radius and the minimum turning radius of the pursuer such that the evader can evade indefinitely. This problem has a lot of variants such as references [3], [4] and [5] to cite a few. In recent past, lot of attention has been received by cooperative control strategies for detection of targets. McLain et al. [6] have addressed the problem of cooperative rendezvous in which multiple UAVs are to arrive simultaneously at their targets. Polycarpou et al. [7] have presented a cooperative target search using online learning and computing guidance trajectories for the agents. Recently, Tang et al. [8], [9] have presented cooperative motion planning methods for multiple UAVs to traverse through slow moving targets and for firstorder mobile sensing agents to detect a moving target that lies in a known initial region respectively. Also, McGee et al. [10] have proposed guaranteed strategies to search for mobile evaders in a plane.

Shaunak D. Bopardikar, Francesco Bullo and João Hespanha are with the Center for Control, Dynamical systems and Computation (CCDC), University of California at Santa Barbara, Santa Barbara, CA 93106, USA, \{shaunak, bullo\}eengineering.ucsb.edu, hespanha@ece.ucsb.edu

\section{B. Contributions}

We address the case when one homicidal chauffeur is not sufficient to capture the evader. We propose a multiple pursuer formation and a novel four-phase, cooperative strategy for the pursuers. In the first two phases of the strategy, we show that the problem is equivalent to having a single pursuer and the task being getting the evader along its direction of motion and at a specified distance, given any set of initial conditions. Next, we show that in the last two phases, the pursuers confine the evader within a bounded region, through which there exists no evader trajectory that avoids capture. We characterize the required number of pursuers and the required value of the evader/pursuers speed ratio for which our strategy is guaranteed to lead to confinement. To the best of our knowledge, this is the first paper that proposes and solves an evader confinement problem. Finally, we characterize a class of confinement strategies and determine a minimum number of pursuers required for any strategy in this class to achieve confinement.

\section{Biological Motivation}

The inspirations for the strategy proposed in this paper have been derived from certain aspects of fish behavior. It has been recently reported by Gazda et al. [11] that in Cedar Key, Florida, USA, individual "driver" dolphins herd slower, more agile prey in circles as well as towards the tightly-grouped "barrier" dolphins. Pitman et al. [12] have reported a herd of killer whales imposing a confinement on pantropical spotted dolphins. The whales cut out up to three dolphins from a school and proceeded to take turns chasing a single dolphin and keeping it within a confined area. These facts give us some hints towards selecting favorable predator formations. The daisy-chain formation that has inspired this work is observed among tarpon fish, as per an informal conversation with biologist Prof. David Skelly, Yale University.

\section{Organization}

The problem assumptions and its mathematical model are presented in Section II. Pursuer formation, the CONFINE strategy and the main results are presented in Section III. The proofs of the main results along with intermediate results required are presented in Section IV. Conclusions and future directions for this work are summarized in Section V.

\section{Problem Set-uP}

Our cooperative homicidal chauffeur game is played in an unbounded, planar environment between a single evader and multiple pursuers. We assume that all the players have unlimited sensing capabilities. The pursuers have identical motion abilities and possess greater speed than that of the evader. However, the evader can make arbitrarily sharp 
turns while the pursuers cannot turn more than a minimum turning radius. We assume that the instantaneous position and velocity of the evader is available to all pursuers.

Let $e(t)$ and $p_{k}(t)$, for $k \in\{1, \ldots, N\}$, denote the positions of the evader and the $k^{t h}$ pursuer in $\mathbb{R}^{2}$ at time $t$, as shown in Figure 1. Let $v_{\mathrm{e}}$ and $v_{\mathrm{p}}$ denote the speeds of the evader and all the pursuers, respectively. Let $\bar{v}_{\mathrm{e}}$ and $\bar{v}_{\mathrm{p}, k}$ denote the velocity vectors of the evader and the $k^{\text {th }}$ pursuer, respectively. Given a minimum turning radius $\rho>0$, the mathematical model for this problem can be described as follows [2].

$$
\text { For evader: } \dot{e}_{x}(t)=v_{\mathrm{e}} \cos \theta_{\mathrm{e}}(t)
$$

$$
\dot{e}_{y}(t)=v_{\mathrm{e}} \sin \theta_{\mathrm{e}}(t)
$$

For pursuers: $\dot{p}_{k, x}(t)=v_{\mathrm{p}} \cos \theta_{\mathrm{p}, k}(t)$,

$$
\begin{aligned}
\dot{p}_{k, y}(t) & =v_{\mathrm{p}} \sin \theta_{\mathrm{p}, k}(t), \\
\dot{\theta}_{\mathrm{p}, k} & =\frac{v_{\mathrm{p}}}{\rho} u_{\mathrm{p}, k},
\end{aligned}
$$

where $\theta_{\mathrm{e}}(t)$ and $\theta_{\mathrm{p}, k}(t)$ are respectively the angles made by the velocity vectors of the evader and of the $k^{t h}$ pursuer with reference to a global $X$ axis. $u_{\mathrm{p}, k}$ is the control applied by the $k^{t h}$ pursuer and satisfies the constraint

$$
\left\|u_{\mathrm{p}, k}\right\| \leq 1
$$

We define the evader/pursuer speed ratio $\gamma:=v_{\mathrm{e}} / v_{\mathrm{p}}$ and assume $\gamma<1$. Given a capture radius $c>0$, the evader is said to be captured if, at some time $t$ and for some $k$,

$$
\left\|p_{k}(t)-e(t)\right\| \leq c
$$

In what follows, without loss of generality, we set the capture radius $c$ and the pursuers speed $v_{\mathrm{e}}$ to 1 . In summary, our cooperative homicidal chauffeur game is described by the number of pursuers $N \in \mathbb{N}$, the minimum turning radius $\rho \in \mathbb{R}_{>0}$, and the evader/pursuers speed ratio $\left.\gamma \in\right] 0,1[$.

Next, we introduce the notion of confinement as follows.

Definition II.1 (Confinement) The evader is said to be confined to a bounded region $\mathcal{G} \subset \mathbb{R}^{2}$ at time $t^{*}$ if $e\left(t^{*}\right) \in \mathcal{G}$ and there exist pursuer trajectories $p_{k}:\left[t^{*},+\infty\left[\rightarrow \mathbb{R}^{2}\right.\right.$ satisfying equation (1) such that the evader cannot leave $\mathcal{G}$ without being captured.

A set of functions $\left\{u_{\mathrm{p}, k}\right\}$, for $k \in\{1, \ldots, N\}$, leading to evader confinement is termed as a confinement strategy. In the case of a single pursuer and single evader, Isaacs [2] has shown that there exists an evasion policy if $\rho>2 /(\pi-2)$ and if the evader/pursuer speed ratio $\gamma \geq \gamma_{\min }(\rho)$, where $\left.\gamma_{\min }:\right] 2 /(\pi-2),+\infty[\rightarrow] 0,1[$ is the unique solution to

$$
\frac{1}{x}=\sqrt{1-\gamma_{\min }(x)^{2}}+\gamma_{\min }(x) \arcsin \left(\gamma_{\min }(x)\right)-1 .
$$

We seek cooperative, deterministic multiple-pursuer strategies which guarantee a confinement of the evader when $\gamma \in\left[\gamma_{\min }(\rho), 1[\right.$ for $\rho>2 /(\pi-2)$.

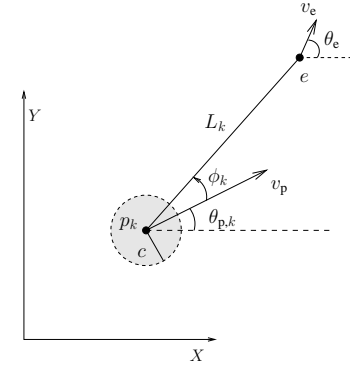

Fig. 1. Variables in the homicidal chauffeur game. The shaded region around the pursuer indicates its capture disc.

\section{The CONFIne Strategy}

In this section, we describe our proposed CONFINE strategy for the pursuers to confine the evader and the corresponding main results. We first propose the following arrangement for the pursuers.

\section{Definition III.1 (Pursuers daisy-chain formation)}

Given an inter-pursuer separation $s_{\text {ip }}>0$, the set $\left\{p_{1}, \ldots, p_{N}, \bar{v}_{\mathrm{p}, 1}, \ldots, \bar{v}_{\mathrm{p}, N}\right\}$ is said to be in a daisy-chain formation if, for every $k \in\{2, \ldots, N\}$, there exists a solution $\eta:\left[0, s_{\mathrm{ip}}\right] \rightarrow \mathbb{R}^{2}$ of equation (1) satisfying

$$
\begin{aligned}
\eta(0) & =p_{k}, & \dot{\eta}(0) & =\bar{v}_{\mathrm{p}, k}, \\
\eta\left(s_{\text {ip }}\right) & =p_{k-1}, & \dot{\eta}\left(s_{\text {ip }}\right) & =\bar{v}_{\mathrm{p}, k-1} .
\end{aligned}
$$

A daisy-chain formation has the following property.

Proposition III.2 (A Daisy-chain property) Assume the pursuers are in a daisy-chain formation with separation $s_{\mathrm{ip}}$. Any feasible path taken by the first pursuer can be exactly traversed by the $k^{\text {th }}$ pursuer, for $k \in\{2, \ldots, N\}$, in the daisy-chain after a time delay of $(k-1) s_{\mathrm{ip}}$.

Figure 2 shows an example of a daisy-chain formation.

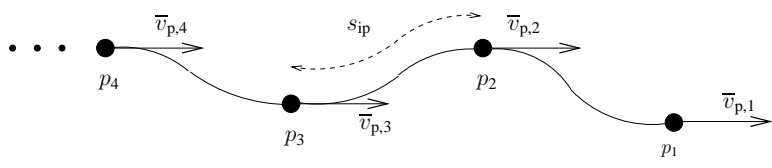

Fig. 2. A daisy-chain formation

Given $\left\{p_{k-1}, p_{k}, \bar{v}_{\mathrm{p}, k-1}, \bar{v}_{\mathrm{p}, k}\right\}$ in a daisy-chain formation with inter-pursuer separation $s_{\text {ip }}$, let $\mathcal{C}_{1}$ and $\mathcal{C}_{2}$ be curves which are tangent to $\mathcal{B}_{1}(\eta(t))$ for every $\left.t \in\right] 0, s_{\text {ip }}$, where for $a \in \mathbb{R}^{2}, \mathcal{B}_{1}(a) \subset \mathbb{R}^{2}$ denotes a circle of radius 1 , centered at $a$. Here, $\eta$ is a curve described in Definition III.1. Then, the evader is said to move between $p_{k-1}$ and $p_{k}$ if there is an evader trajectory from $\mathcal{C}_{1}$ to $\mathcal{C}_{2}$ (or vice-versa).

Given the pursuers' minimum turning radius $\rho$, for the evader/pursuers speed ratio $\gamma \leq 1-\frac{1}{\rho}$, we define critical inter-pursuer separation $s_{\mathrm{ip}}^{*}(\gamma, \rho)$ as follows: if $r_{1}^{2}:=(1+$ 
$\left.\rho^{2}\right)+2 \rho \sqrt{1-\gamma^{2}}, r_{2}^{2}:=\left(1+\rho^{2}\right)-2 \rho \sqrt{1-\gamma^{2}}$, and

$$
\begin{aligned}
\Delta \theta:=\arctan & \left(\frac{1-\rho \sqrt{1-\gamma^{2}}}{\gamma \rho}\right) \\
& -\arctan \left(\frac{1+\rho \sqrt{1-\gamma^{2}}}{\gamma \rho}\right)+\frac{2 \sqrt{1-\gamma^{2}}}{\gamma},
\end{aligned}
$$

then

$$
s_{\mathrm{ip}}^{*}(\gamma, \rho):=\rho\left(\Delta \theta+\arcsin \frac{\gamma}{r_{1}}+\arcsin \frac{\gamma}{r_{2}}\right) .
$$

This $s_{\mathrm{ip}}^{*}(\gamma, \rho)$ has the following property, whose proof is presented in Section IV.

\section{Lemma III.3 (Property of $s_{\text {ip }}^{*}(\gamma, \rho)$ ) Given}

$\left\{p_{k-1}, p_{k}, \bar{v}_{\mathrm{p}, k-1}, \bar{v}_{\mathrm{p}, k}\right\} \quad$ in a daisy-chain formation and the evader/pursuers speed ratio satisfying $\gamma \leq 1-\frac{1}{\rho}$, to prevent an evader from moving between $p_{k-1}$ and $p_{k}$ without being captured, the inter-pursuer separation must not exceed $s_{\mathrm{ip}}^{*}(\gamma, \rho)$.

Given a point $p$ and a unit vector $x \in \mathbb{R}^{2}$, we define the region of confinement $\mathcal{G}_{\mathrm{c}}(p, x) \subset \mathbb{R}^{2}$ for the CONFINE strategy as follows: choose $a, b, c \in \mathbb{R}^{2}$ such that

(i) $x$ rotated counter-clockwise about $p$ by $\frac{\pi}{2}$ becomes parallel to $(a-p),(p-c) \| x$ and,

(ii) $a, b, c, p$ are vertices of a rectangle such that $\|a-b\|=$ $\|p-c\|=\frac{2 \pi \gamma \rho}{1-\gamma}$ and $\|a-p\|=\|b-c\|=\rho+1$, as shown in Figure 3.

Let $\operatorname{Rect}(a, b, c, p) \subset \mathbb{R}^{2}$ denote the rectangular region formed by the four points $a, b, c$ and $p$. Then, $\mathcal{G}_{\mathrm{c}}(p, x) \subset \mathbb{R}^{2}$ is defined as interior of the union of $\mathcal{B}_{\rho+1}\left(\frac{a+p}{2}\right), \mathcal{B}_{\rho+1}\left(\frac{b+c}{2}\right)$ and $\operatorname{Rect}(a, b, c, p)$.

We define a point $a \in \mathbb{R}^{2}$ to be aligned with the $k^{\text {th }}$ pursuer if $\bar{v}_{\mathrm{p}, k} \|\left(a-p_{k}\right)$.

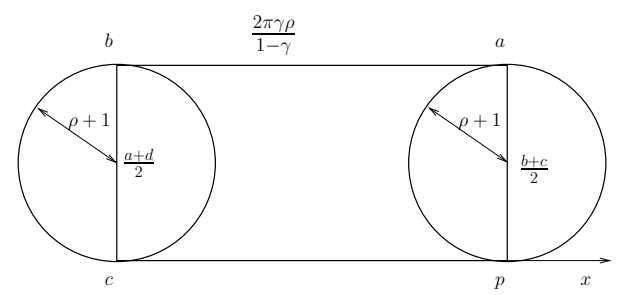

Fig. 3. Defining the region of confinement, $\mathcal{G}_{\mathrm{c}}$ for the CONFINE strategy

We now describe the CONFINE strategy. Let the evader be located at $e(0)$, as shown in Figure 4. Let $\left\{p_{1}, \ldots, p_{N}, \bar{v}_{\mathrm{p}, 1}, \ldots, \bar{v}_{\mathrm{p}, N}\right\}$ be a daisy-chain formation with $s_{\text {ip }}=s_{\text {ip }}^{*}(\gamma, \rho)$ and the first pursuer at $p_{1}(0)$. Let the angle between $\bar{v}_{\mathrm{p}, 1}(0)$ and $\left(e(0)-p_{1}(0)\right)$ be $\phi_{0}$ and $\| p_{1}(0)-$ $e(0) \|=L_{0}$. Due to Proposition III.2, it suffices to specify the strategy for $p_{1}$. The CONFINE strategy is as follows.

(i) Pre-Align phase: This phase is needed if $e(0)$ is not aligned with $p_{1}(0)$ or if $L_{0}<(1+\gamma) l_{\mathrm{st}}$, where $l_{\mathrm{st}} \triangleq \frac{2 \pi \gamma \rho}{1-\gamma}$. Let $l_{\mathrm{p}}$ be the minimum of the roots of the quadratic equation:

$$
\begin{aligned}
\left(\gamma\left(l_{\mathrm{p}}+2 \pi \rho\right)\right. & \left.+(1+\gamma) l_{\mathrm{st}}+\rho\right)^{2} \\
& =\left(l_{\mathrm{p}}-L_{0} \cos \psi_{0}\right)^{2}+\left(\rho-L_{0} \sin \psi_{0}\right)^{2} .
\end{aligned}
$$

$p_{1}$ moves on a straight line path of length $l_{\mathrm{p}}$ and then moves on a circle of radius $\rho$ and center on the side not containing $e(0)$ of the line along $\bar{v}_{\mathrm{p}, 1}\left(l_{\mathrm{p}}\right)$. If $\phi_{0}=0$ or $\pi$, then the center of the circle of radius $\rho$ can be chosen to be on either side of the line along $\bar{v}_{\mathrm{p}, 1}\left(l_{\mathrm{p}}\right)$.

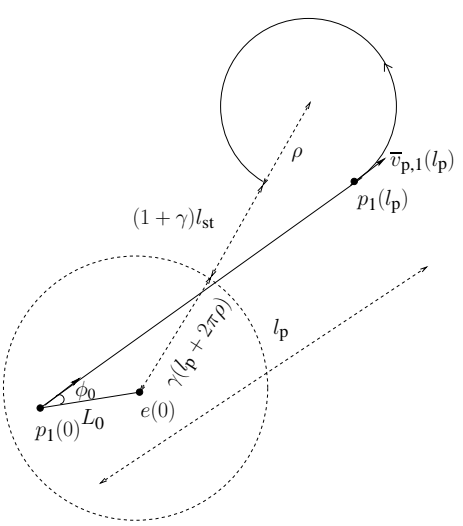

Fig. 4. The Pre-Align phase: the bold line shows the trajectory followed by $p_{1}$.

(ii) Align phase: $p_{1}$ moves with the following control law:

$$
u_{\mathrm{p}, 1}\left(\theta_{\mathrm{e}}, e, \theta_{\mathrm{p}, 1}, p_{1}\right)=\frac{\rho \gamma}{\left\|p_{1}-e\right\|} \sin \left(\theta_{\mathrm{e}}-\theta_{\mathrm{p}, 1}\right)
$$

until $\left\|p_{1}-e\right\|=(1+\gamma) l_{\mathrm{st}}$.

(iii) Chase phase: $p_{1}$ moves on a straight line path until either it captures the evader or $\bar{v}_{\mathrm{p}, 1} \perp\left(e-p_{1}\right)$, where the symbol $\perp$ denotes perpendicularity. This is shown in Figure 5. Let $t_{\text {chase }}$ denote the time at the end of the Chase phase.

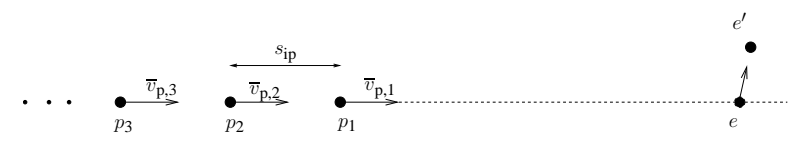

Fig. 5. The Chase phase

(iv) Close phase: First, $p_{1}$ moves on a circle of radius $\rho$ and center $O$ located on the evader side of the line along $\bar{v}_{\mathrm{p}, 1}$, until it covers a distance $\pi \rho$. Second, $p_{1}$ moves on a straight line path of length $l_{\mathrm{st}}$. Third, $p_{1}$ moves on a circle of radius $\rho$ and center $O^{\prime}$ located on the evader side of the line along $\bar{v}_{\mathrm{p}, 1}$, until it covers a distance $\pi \rho$. This motion is shown in Figure 6.

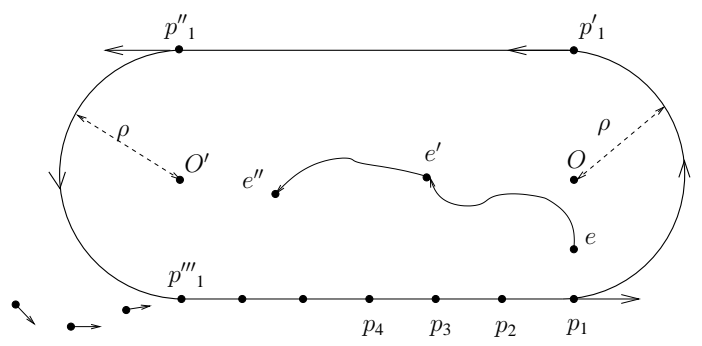

Fig. 6. The Close phase

(v) Final phase: $p_{1}$ moves on the boundary of $\mathcal{G}_{\mathrm{c}}\left(p_{1}\left(t_{\text {chase }}\right), \bar{v}_{\mathrm{p}, 1}\left(t_{\text {chase }}\right)\right)$. 
For every $k \in\{2, \ldots, N\}, p_{k}$ moves on curve $\eta$ described in Definition III.1 for time $t \leq s_{\mathrm{ip}}$. For $t>s_{\mathrm{ip}}, u_{\mathrm{p}, k}(t)=$ $u_{\mathrm{p}, k-1}\left(t-s_{\text {ip }}\right)$.

This strategy gives us the following result.

Theorem III.4 (Confinement) Consider a cooperative homicidal chauffeur game with parameters $N \in \mathbb{N}$, $\rho>\frac{2}{\pi-2}$, and $\left.\gamma \in\right] 0,1\left[\right.$. Let $t_{\text {chase }}$ denote the time at the end of the Chase phase. The CONFINE strategy guarantees confinement of the evader inside the region $\mathcal{G}_{\mathrm{c}}\left(p_{1}\left(t_{\text {chase }}\right), \bar{v}_{\mathrm{p}, 1}\left(t_{\text {chase }}\right)\right)$ at time $t_{\text {chase }}$ if

$$
N \geq N_{\min }:=\left\lceil\frac{2 \pi \rho(1+\gamma)}{s_{\mathrm{ip}}^{*}(\gamma, \rho)(1-\gamma)}\right\rceil,
$$

and if

$$
\gamma \in\left[\gamma_{\min }(\rho), \gamma_{\max }(\rho)\right],
$$

where $\gamma_{\max }(\rho)$ is the smallest root in the interval $] 0,1[$ of

$$
\left(1-\gamma^{2}\right)\left(2-\frac{2 \pi \gamma^{2}}{(1-\gamma)} \sqrt{\frac{1+\gamma}{1-\gamma}}+\frac{1}{\rho}\right)^{2}=\pi^{2} \gamma^{2} .
$$

Moreover, if $\gamma>\gamma_{\max }(\rho)$ or if $N<N_{\min }$, then the evader can escape the region $\mathcal{G}_{\mathrm{c}}\left(p_{1}\left(t_{\text {chase }}\right), \bar{v}_{\mathrm{p}, 1}\left(t_{\text {chase }}\right)\right)$.

Remark III.5 Because

$$
\lim _{\rho \rightarrow+\infty} \gamma_{\min }(\rho)=0^{+}, \lim _{\rho \rightarrow+\infty} \gamma_{\max }(\rho) \approx .293,
$$

for sufficiently large values of $\rho$, the interval $\left[\gamma_{\min }(\rho), \gamma_{\max }(\rho)\right]$ is non-empty.

Remark III.6 (From confinement to capture) If a sufficiently large number of pursuers is available, then capture can be achieved in a final maneuver as follows: $N_{\text {min }}$ pursuers confine the evader, while some additional pursuers arrange themselves in a line as shown in Figure 7 and move simultaneously through $\mathcal{G}_{\mathrm{c}}\left(p_{1}\left(t_{\text {chase }}\right), \bar{v}_{\mathrm{p}, 1}\left(t_{\text {chase }}\right)\right)$.

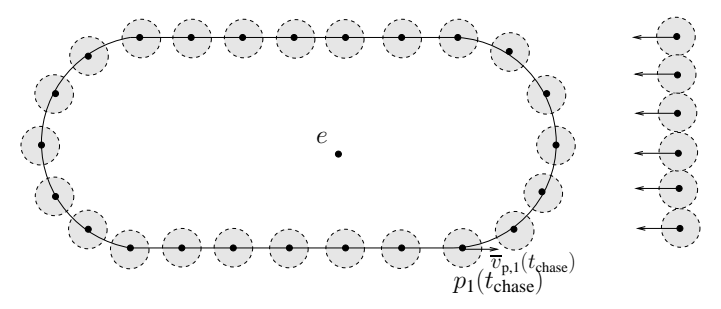

Fig. 7. Achieving capture with a sufficiently large number of pursuers

We now define a class of confinement strategies as follows.

Definition III.7 (Daisy chain-based strategy) A confinement strategy is termed as daisy chain-based if, using that strategy,

(i) the evader is confined to a region $\mathcal{G}$ at time $t^{*}$,

(ii) the set $\left\{p_{1}\left(t^{*}\right), \ldots, p_{N}\left(t^{*}\right), \bar{v}_{\mathrm{p}, 1}\left(t^{*}\right), \ldots, \bar{v}_{\mathrm{p}, N}\left(t^{*}\right)\right\}$ is a daisy-chain formation with inter-pursuer separation $s_{\text {ip }} \leq s_{\mathrm{ip}}^{*}(\gamma, \rho)$ and, (iii) the pursuer trajectories $p_{k}:\left[t^{*},+\infty\left[\rightarrow \mathbb{R}^{2}, k \in\right.\right.$ $\{2, \ldots, N\}$ satisfy

$$
u_{\mathrm{p}, k}(t)=u_{\mathrm{p}, k-1}\left(t-s_{\mathrm{ip}}\right), \quad \text { for } t \geq t^{*} .
$$

Clearly, the CONFINE strategy is a daisy chain-based strategy. We now have the following result.

Theorem III.8 (Minimum number of pursuers) Let $\rho>$ $\frac{2}{\pi-2}$ and the evader/pursuers speed ratio $\gamma \geq \gamma_{\min }(\rho)$, where $\gamma_{\min }(\rho)$ is the solution to equation (2). Then, to achieve confinement using any daisy chain-based strategy, the number of pursuers must be at least $N^{*}=\left\lceil\frac{2 \pi \rho}{s_{\mathrm{ip}}^{*}(\gamma, \rho)}\right\rceil$.

\section{Proofs of the Main Results}

In this section, we prove the main results from Section III. We begin with certain intermediate results towards proving the main results. For the want of space, we provide the sketch of the proofs to Lemmas III.3, IV.3 and IV.5. We refer the reader to the report [13] for the complete proofs.

We say that a daisy-chain with separation $s_{\text {ip }}$ is closed if for some $p_{k}(k \neq 1)$, there exists a $t_{k} \leq s_{\text {ip }}$ and a solution $\eta:\left[0, t_{k}\right] \rightarrow \mathbb{R}^{2}$ of equation (1) satisfying

$$
\begin{aligned}
\eta(0) & =p_{1}, & \dot{\eta}(0) & =\bar{v}_{\mathrm{p}, 1}, \\
\eta\left(t_{k}\right) & =p_{k}, & \dot{\eta}\left(t_{k}\right) & =\bar{v}_{\mathrm{p}, k},
\end{aligned}
$$

such that the evader cannot move between $p_{1}$ and $p_{k}$ without being captured. We first sketch the proof of Lemma III.3.

Proof sketch of Lemma III.3: As shown in Figure 8, we consider the evader's motion in a reference frame attached to the center $O$ of the circle of radius $\rho$ through pursuers $p_{k-1}$ and $p_{k}$ and rotating with angular speed $\frac{1}{\rho}$ in the direction of pursuer motion. We show that if the interpursuer separation does not exceed $s_{\mathrm{ip}}^{*}(\gamma, \rho)$, then any evader trajectory from arc $U W$ to $P Q$ must enter the capture ball of pursuer $p_{k-1}$ or $p_{k}$. We compute the analytical expression for $s_{\mathrm{ip}}^{*}(\gamma, \rho)$ by determining the optimal trajectory for the evader in polar coordinates with origin at $O$ that maximizes the radial distance covered by the evader for a given angular displacement.

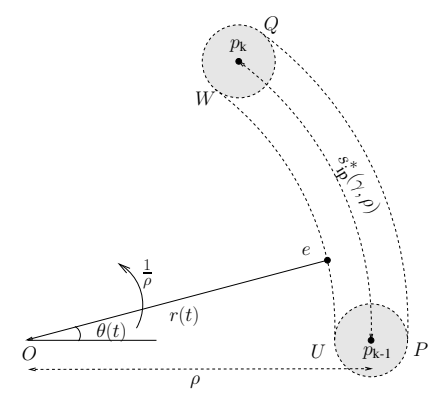

Fig. 8. Illustrating proof sketch of Lemma IV.1. The shaded regions are the capture discs of $p_{k-1}$ and $p_{k}$.

We have the following result for the CONFINE strategy.

Lemma IV.1 (A CONFINE strategy property) For the CONFINE strategy, the evader cannot move without being captured between pursuers $p_{k}$ and $p_{k-1}$, for all 
$k \in\{2, \ldots, N\}$ and between any two consecutive pursuers if the daisy-chain is closed.

Proof: We show that the inter-pursuer spacing, $s_{\text {ip }}=$ $s_{\text {ip }}^{*}(\gamma, \rho)$ is actually necessary in the CoNFINE strategy to prevent the evader from escaping between any two pursuers $p_{k-1}$ and $p_{k}$ and between any two pursuers when the daisychain is closed. It is known from [10] that when $p_{k-1}$ is aligned with $p_{k}$, the inter-pursuer separation $s_{\text {ip }}=\frac{2}{\gamma}$ necessary and sufficient so that the evader cannot move between them without being captured.

Using equation (3), it can be easily verified that for a given $\gamma, s_{\text {ip }}^{*}(\gamma, \rho)$ increases monotonically with $\rho$ and as $\rho \rightarrow \infty$, $s_{\text {ip }}^{*}(\gamma, \rho) \rightarrow \frac{2}{\gamma}$. Thus, the necessary spacing for the CONFINE strategy is $s_{\text {ip }}^{*}(\gamma, \rho)$.

We now prove the following property of the Pre-Align phase.

Lemma IV.2 (Pre-Align phase) The Pre-Align phase terminates in finite time with the evader aligned with $p_{1}$ at a distance greater than $(1+\gamma) l_{\mathrm{st}}$.

Proof: The total time taken by $p_{1}$ to cover a distance $l_{\mathrm{p}}$ followed by distance of $2 \pi \rho$ is $\left(l_{\mathrm{p}}+2 \pi \rho\right)$. In that time, the evader's reachability set is the dotted circle of radius $\gamma\left(l_{\mathrm{p}}+2 \pi \rho\right)$, centered at $e(0)$, as shown in Figure 4. Thus, to compute $l_{\mathrm{p}}$, we impose the condition that the minimum distance between the evader's reachability set and the circular portion of the path of $p_{1}$ must be $(1+\gamma) l_{\text {st }}$. Using elementary geometry, the equation (4) for $l_{\mathrm{p}}$ follows.

We now prove the following property of the Align phase.

Lemma IV.3 (Align phase) The Align phase of CONFINE strategy terminates after finite time with the evader aligned with $p_{1}$ and $\left\|p_{1}-e\right\|=(1+\gamma) l_{\mathrm{st}}$.

Proof sketch of Lemma IV.3: Consider the system as shown in Figure 1 with $k=1$. At the end of the Pre-align phase, angle $\phi=0$. Using the control law for Align phase, we show that $\phi=0$, for all subsequent time instants as long as distance $L(t) \geq \gamma \rho$.

We now obtain an upper-bound for the distance between the first pursuer and evader at the end of the Chase phase.

Lemma IV.4 (Upper bound on $d$ ) Let $d$ denote the distance $\left\|p_{1}-e\right\|$ at the end of the Chase phase. Then,

$$
d \leq \gamma \sqrt{\frac{1+\gamma}{1-\gamma}} l_{\mathrm{st}}
$$

or, equivalently, for $\mu \triangleq \frac{d-1}{\rho}$ and $l_{\mathrm{st}}=\frac{2 \pi \gamma \rho}{1-\gamma}$,

$$
\mu \leq \frac{2 \pi \gamma^{2}}{(1-\gamma)} \sqrt{\frac{1+\gamma}{1-\gamma}}-\frac{1}{\rho}
$$

Proof: We have the evader aligned with the pursuer at a distance $(1+\gamma) l_{\text {st }}$ as shown in Figure 9. Let the evader strike out at an angle $\psi$ as shown. So at the end of the Chase phase, it follows from trigonometry that

$$
d=\frac{(1+\gamma) l_{\mathrm{st}} \sin \psi}{\left(\frac{1}{\gamma}-\cos \psi\right)}
$$

Thus, $d$ is a maximum when $\cos \psi=\gamma$ and the result follows.

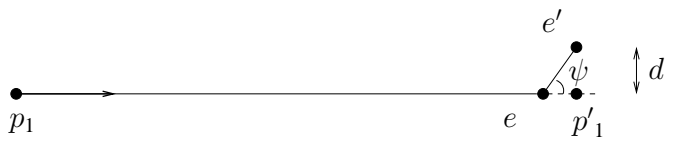

Fig. 9. Illustrating proof of Lemma IV.4

The right hand side of equation (6) can be verified to be positive and monotonic increasing with $\gamma$ for $\gamma \geq \gamma_{\min }(\rho)$. We now establish an upper bound for the speed ratio $\gamma$ so that the evader always remains on the same side of the line along velocity vector of $p_{1}$.

Lemma IV.5 (Upper bound on $\gamma$ ) For the CONFINE strategy to succeed, the evader/pursuers speed ratio $\gamma$ must satisfy,

$$
\gamma \leq \frac{2-\mu}{\sqrt{\pi^{2}+(2-\mu)^{2}}}, \text { where } \mu=\frac{d-1}{\rho} .
$$

Proof sketch of Lemma IV.5: We show that for any angle $\omega \in\left[-\frac{\pi}{2}, \frac{\pi}{2}\right]$ shown in Figure 10 and to guarantee escape against the pursuer strategy, the evader needs to reach point $B^{\prime}$ before the pursuer reaches $B$. This gives us the required upper bound on $\gamma$.

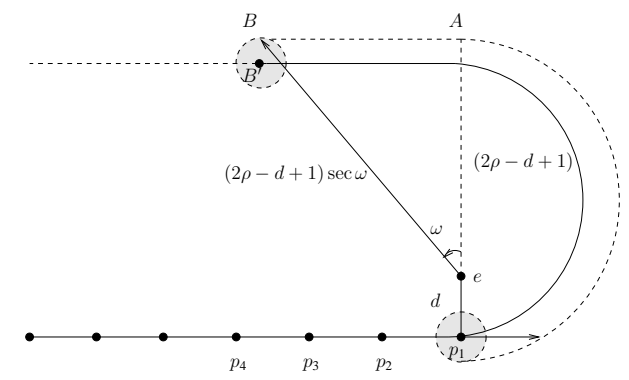

Fig. 10. Illustrating proof of Lemma IV.5. The shaded region about $B^{\prime}$ indicates capture region of $p_{1}$ when it reaches $B^{\prime}$

We are now ready to prove Theorem III.4.

Proof of Theorem III.4:

The Chase phase of the strategy ensures that a length of at least $l_{\mathrm{st}}$ is a straight segment. For the Close phase to guarantee evader confinement, it suffices that the daisy chain is closed at the end of the Close phase. This final configuration is illustrated in Figure 11. Thus, by the time

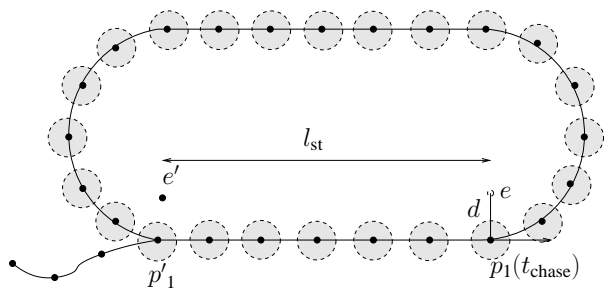

Fig. 11. Closing of the daisy-chain

the evader covers at most a distance $l_{\mathrm{st}}$ in the Close phase, 
$p_{1}$ must cover a total length of $2 \pi \rho+l_{\mathrm{st}}$, which gives us the following condition on $l_{\mathrm{st}}$ :

$$
\frac{l_{\mathrm{st}}}{\gamma} \geq 2 \pi \rho+l_{\mathrm{st}} \quad \Longrightarrow \quad l_{\mathrm{st}} \geq \frac{2 \pi \gamma \rho}{1-\gamma} .
$$

So we let $l_{\mathrm{st}}=\frac{2 \pi \gamma \rho}{1-\gamma}$. This justifies our definition of the quantity $l_{\text {st }}$ throughout the strategy. Using Lemma IV.5, we seek to determine the minimum over the set of all $\mu$ that satisfy equation (6) . This gives us a uniform upper-bound $\gamma_{\max }$. Thus,

$$
\gamma_{\max }=\min _{\mu} \frac{2-\mu}{\sqrt{\pi^{2}+(2-\mu)^{2}}} .
$$

But the function on the right-hand side is monotonic decreasing with $\mu$. The maximum value of $\mu$ is achieved when equality holds in equation (6), since the right-hand side of equation (6) is a monotonic increasing function of $\gamma$. Thus, the upper bound on speed ratio $\gamma_{\max }(\rho)$ is obtained by solving equation (5) which upon simplification gives a polynomial equation in $\gamma$ and $\gamma_{\max }(\rho)$ is its smallest root in the interval ] $0,1[$.

A sufficient number of pursuers for the strategy CONFINE to succeed is obtained by determining the total distance covered by $p_{1}$ in the Close phase added to $l_{\text {st }}$, i.e., $2\left(l_{\mathrm{st}}+\right.$ $\pi \rho)=\frac{2 \pi(1+\gamma) \rho}{(1-\gamma)}$. Thus, the number of pursuers needed is at least $N_{\min }:=\left[\frac{2 \pi(1+\gamma) \rho}{(1-\gamma) s_{\mathrm{ip}}^{*}(\gamma, \rho)}\right]$. Since the daisy-chain is closed at the end of Close phase and there is no escape trajectory between any two consecutive pursuers, it is clear that the evader is confined to $\mathcal{G}_{\mathrm{c}}\left(p_{1}\left(t_{\text {chase }}\right), \bar{v}_{\mathrm{p}, 1}\left(t_{\text {chase }}\right)\right)$.

Moreover, if $\gamma>\gamma_{\max }(\rho)$, then there exists an evader trajectory that leaves $\mathcal{G}_{\mathrm{c}}\left(p_{1}\left(t_{\text {chase }}\right), \bar{v}_{\mathrm{p}, 1}\left(t_{\text {chase }}\right)\right)$ as seen in the proof of Lemma IV.5. Also, if the number of pursuers $N<N_{\min }$, then the daisy chain is not closed at the end of the Close phase. Thus, there exists an initial condition and a corresponding evader strategy so that it can leave $\mathcal{G}_{\mathrm{c}}\left(p_{1}\left(t_{\text {chase }}\right), \bar{v}_{\mathrm{p}, 1}\left(t_{\text {chase }}\right)\right)$ by moving between $p_{1}$ and $p_{N}$. This completes the proof.

Proof of Theorem III.8:

Let the evader be confined to a region $\mathcal{G}$ at time $t^{*}$. Due to part (iii) of Definition III.7, daisy chain formation is maintained at all times $t \geq t^{*}$ and the boundary of region $\mathcal{G}$ must be a continuous, closed curve with radius of curvature bounded below by $\rho$. To minimize the number of pursuers, the pursuers need to be placed on the smallest possible region having this property, which is a circle of radius $\rho$. From Lemma IV.1, we deduce that the inter-pursuer spacing $s_{\text {ip }}=s_{\text {ip }}^{*}(\gamma, \rho)$ is necessary to ensure that the evader cannot move between any two successive pursuers in the daisychain formation, without being captured. Thus, the number of pursuers must be at least $N^{*}=\left\lceil\frac{2 \pi \rho}{s_{\mathrm{ip}}^{*}(\gamma, \rho)}\right\rceil$.

\section{COnClusions And Future Directions}

We addressed a cooperative homicidal chauffeur game in which a single pursuer is unable to capture an evader, given an arbitrary initial condition. We proposed a bioinspired cooperative multiple pursuer strategy that guarantees confinement of an evader to a bounded region, from any initial condition. We characterized the required number of pursuers and the required value of the evader/pursuers speed ratio for which our CONFINE strategy is guaranteed to lead to confinement. We also characterized a class of confinement strategies and determined a minimum number of pursuers needed for any strategy in that class to achieve confinement.

Future research will consider how to determine alternate strategies possibly involving multiple maneuvers to achieve confinement. It would also be interesting to know the minimum number of pursuers required for any confinement strategy.

\section{ACKNOWLEDGMENTS}

This material is based upon work supported in part by ARO MURI Award W911NF-05-1-0219 and ONR Award N00014-07-1-0721 and by the Institute for Collaborative Biotechnologies through the grant DAAD19-03-D-0004 from the U.S. Army Research Office. The authors would like to thank Prof. David Skelly for insightful conversations about animal behavior.

\section{REFERENCES}

[1] R. Isaacs, Differential games. New York: John Wiley, 1965.

[2] T. Başar and G. J. Olsder, Dynamic Noncooperative Game Theory, 2nd ed. Philadelphia, PA: SIAM, 1999.

[3] V. Y. Glizer, "Homicidal chauffeur game with target set in the shape of a circular angular sector: Conditions for existence of a closed barrier," Journal of Optimization Theory \& Applications, vol. 101, no. 3, pp. 581-598, 1999.

[4] M. Pachter and Y. Yavin, "A stochastic homicidal chauffeur pursuitevasion differential game," Journal of Optimization Theory \& Applications, vol. 34, no. 3, pp. 405-424, 1981.

[5] W. M. Getz and M. Pachter, "Two-target pursuit-evasion differential games in the plane," Journal of Optimization Theory \& Applications, vol. 34, no. 3, pp. 383-403, 1981.

[6] T. W. McLain, P. R. Chandler, S. Rasmussen, and M. Pachter, "Cooperative control of UAV rendezvous," in American Control Conference, Arlington, VA, June 2001, pp. 2309-2314.

[7] M. M. Polycarpou, Y. Yang, and K. M. Passino, "A cooperative search framework for distributed agents," in IEEE International Symposium on Intelligent Control, Mexico City, Mexico, Sept. 2001, pp. 1-6.

[8] Z. Tang and Ü. Özgüner, "Motion planning for multi-target surveillance with mobile sensor agents," IEEE Transactions on Robotics, vol. 21 , no. 5, pp. 898-908, 2005.

[9] _ - "On non-escape search for a moving target by multiple mobile sensor agents," in American Control Conference, Minneapolis, Minnesota, USA, June 2006, pp. 3525-3530.

[10] T. G. McGee and J. K. Hedrick, "Guaranteed strategies to search for mobile evaders in the plane," in American Control Conference, Minneapolis, MN, June 2006, pp. 2819-2824.

[11] S. K. Gazda, R. C. Connor, R. K. Edgar, and F. Cox, "A division of labour with role specialization in group-hunting bottlenose dolphins (Tursiops truncatus) off Cedar Key, Florida," Proceedings of the Royal Society B: Biological Sciences, vol. 272, no. 1559, pp. 135-140, 2005.

[12] R. L. Pitman, S. O'Sullivan, and B. Mase, "Killer whales (Ornicus orca) attack a school of pantropical spotted dolphins (Stenella attenuata) in Gulf of Mexico," Aquatic Mammals, vol. 29, no. 3, pp. 321324, 2003.

[13] S. D. Bopardikar, F. Bullo, and J. P. Hespanha, "A cooperative homicidal chauffeur game," Center for Control, Dynamical Systems and Computation. University of California at Santa Barbara, Tech. Rep. CCDC-07-0827, 2007, available electronically at http: //ccdc. mee.ucsb.edu. 